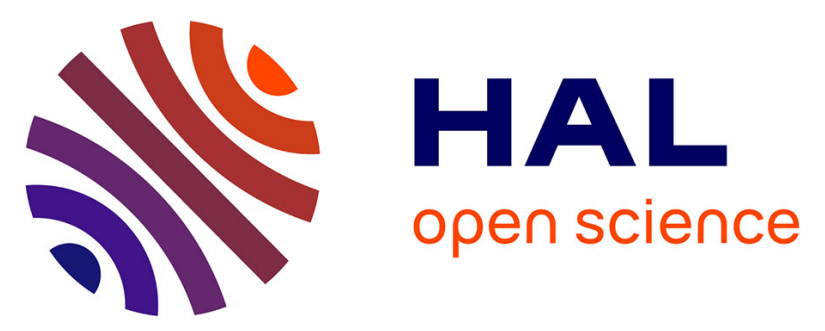

\title{
La traducción de los verbos de postura del neerlandés al español: un análisis comparativo de los verbos de localización haber y tener
}

Machteld Meulleman, Linde van den Broeck

\section{- To cite this version:}

Machteld Meulleman, Linde van den Broeck. La traducción de los verbos de postura del neerlandés al español: un análisis comparativo de los verbos de localización haber y tener. Romanistisches Jahrbuch, 2012, 63 (1), pp.287-305. 10.1515/roma.63.11 . hal-01617628

\section{HAL Id: hal-01617628 \\ https://hal.science/hal-01617628}

Submitted on 16 Oct 2017

HAL is a multi-disciplinary open access archive for the deposit and dissemination of scientific research documents, whether they are published or not. The documents may come from teaching and research institutions in France or abroad, or from public or private research centers.
L'archive ouverte pluridisciplinaire HAL, est destinée au dépôt et à la diffusion de documents scientifiques de niveau recherche, publiés ou non, émanant des établissements d'enseignement et de recherche français ou étrangers, des laboratoires publics ou privés. 


\title{
La traducción de los verbos de postura del neerlandés al español: un análisis comparativo de los verbos de localización haber y tener
}

Por Machteld Meulleman y Linde Van den Broeck

\begin{abstract}
This study examines empirically how the three main posture verbs in Dutch, i. e. liggen ('to lie'), zitten ('to sit') and staan ('to stand'), are translated into Spanish in presentational contexts. From the analysis of a translation corpus, it turns out that even though the most common translation of the three posture verbs is haber ('there be'), its frequency decreases considerably in favor of tener ('to have') in the case of zitten. Based on a detailed analysis of their respective semantic and pragmatic properties, it is argued that tener appears as soon as there is an «impact » relation between the object and its location, whereas haber expresses genuine containment. Close analysis reveals that it is chiefly the categorical nature of tener that triggers its use as a translation of zitten.
\end{abstract}

\section{Introducción}

Como es bien sabido, una de las diferencias tipológicas más notables entre las lenguas románicas y las germánicas consiste en que las primeras forman parte de las llamadas verb-framed languages y las segundas de las satellite-framed languages (Talmy 2000). La diferencia entre estas dos categorías de lenguas se observa particularmente bien en los verbos (V) de movimiento. Así, como está ilustrado en (1), el español expresa la dirección del movimiento dentro del V (salir) y el modo en un complemento satélite (el gerundio corriendo). Al contrario, en neerlandés el lexema verbal explicita el modo (como lopen 'correr' en (2)), mientras que la dirección queda expresada por un elemento satélite (en este caso el sintagma preposicional (SP) naar buiten).

(1) Salió corriendo.

(2) Hij liep naar buiten.

'El corrió hacia fuera.'

Esta diferencia tipológica se observa también en los V de localización (i. a. Lemmens 2005). Así, paralelamente, las lenguas románicas suelen expresar los conceptos de existencia y de localización con V semánticamente neutros en cuanto al modo de localización como haber y estar en español. En cambio, las lenguas germánicas tienden a especificar la posición física del referente utilizando un $\mathrm{V}$ de postura como liggen ('estar tendido'), staan ('estar de pie') y zitten ('estar sentado') en neerlandés. En su uso literal, estos V indican la postura física de personas (3), pero muchas veces se utilizan metafóricamente para situar objetos (4) e incluso ideas abstractas (5). De hecho, el uso de la expresión semánticamente neutra con er zijn ('haber', cf. 'there be' en inglés) resulta muchas veces inaceptable en neerlandés (6). Huelga decir que en español el uso de $\mathrm{V}$ de postura en muchos de estos contextos resultaría igualmente inaceptable.

(3) Er staat / ligt / zit een man in de tuin.

'Un hombre está de pie / está tendido / está sentado en el jardín.'

(4) Op die tafel ligt een boek dat je moet lezen.

'?En esa mesa está tendido un libro que tienes que leer.'

(5) In deze tekst zitten verschillende ideeën. 
'*En este texto están sentadas varias ideas.'

(6) *Er zijn veel boeken op tafel.

'Hay muchos libros en la mesa.'

Si bien se ha dedicado mucha atención a los $\mathrm{V}$ de movimiento en las verb-framed y satellite-framed languages, Ameka / Levinson (2007) señalan con razón que son muy escasos los estudios sobre la tipología de los V de localización. Suelen concentrarse o bien en el uso de estos V de postura en un idioma particular (como el alemán, el neerlandés, el sueco, etc.) o bien en las diferencias existentes a este respecto entre lenguas de la misma familia ${ }^{1}$.

En la presente contribución entendemos examinar si (y cómo) una lengua románica, el español, expresa los conceptos vinculados con los V de postura de una lengua germánica, el neerlandés. Para lograr este objetivo, investigaremos en base a un análisis empírico de un corpus de traducción del neerlandés al español a) cuáles son las estructuras utilizadas en la traducción española, y b) cómo y en qué medida las diferentes alternativas de traducción reflejan el abanico de matices semánticos expresados por los $\mathrm{V}$ de postura en la versión original neerlandesa ${ }^{2}$.

Ahora bien, en el ámbito de la lingüística hispánica, los estudios sobre los $\mathrm{V}$ de localización suelen centrarse en la diferencia pragmático-discursiva que existe entre haber y estar. Así, haber aparece en construcciones existenciales-presentativas téticas, mientras que estar suele usarse en construcciones locativas categóricas (i. a. Bull 1943). Visto que nuestro objetivo es en primer lugar de índole semántica, nos limitaremos dentro de este trabajo al estudio de los $\mathrm{V}$ de postura en un contexto discursivo particular. Dado el fuerte lazo semántico-cognitivo entre existencia y presentación (Lambrecht 1994), las construcciones presentativas nos parecen el contexto semántico-discursivo por excelencia para alcanzar nuestros objetivos ${ }^{3}$.

A continuación presentamos nuestro análisis empírico. Primero, presentamos una visión de conjunto de las diferentes alternativas de traducción de los tres $\mathrm{V}$ neerlandeses en el corpus español. Luego, nos fijaremos en el uso de los dos $\mathrm{V}$ más frecuentes en el corpus traducido, o sea haber y tener. Finalmente, propondremos una interpretación funcional cognitiva del reparto entre ambos V.

\section{Alternativas de traducción}

Si averiguamos cuáles son los diferentes $\mathrm{V}$ que ocurren en la traducción española, constatamos - como era de esperar - que los casos de traducción literal son muy escasos, mientras que el uso de haber

\footnotetext{
${ }^{1}$ El uso polisémico de los $\mathrm{V}$ de postura presenta bastante variación interlingüística. Dentro de las lenguas germánicas, véase $i$. a. Newman (2002) para el inglés, Serra Borneto (1996) para el alemán y Lemmens (2002, 2005) para el neerlandés.

${ }^{2}$ Para realizar este estudio, establecimos un corpus de 15 novelas neerlandesas contemporáneas (once novelas de ficción para adultos, tres novelas de literatura juvenil y un diario) y sus respectivas traducciones al español. En cuanto a la variedad neerlandesa estudiada, 12 novelas representan la variedad holandesa y 3 la flamenca. En total, este corpus contiene 134 ocurrencias de er staan, 180 ocurrencias de er zitten y 80 ocurrencias de er liggen.

${ }^{3}$ Lambrecht / Polinsky (1997) muestran que existe una estrategia gramatical universal para marcar los enunciados téticos, o sea enteramente remáticos, poniendo el SN introducido en posición posverbal, típica del OD. Muchas veces figura un complemento locativo en posición preverbal que sirve de marco temático (Kuno 1972, Conti Jiménez 2005). En neerlandés, de no ser así, aparece muchas veces el pronombre adverbial er (cf. there en inglés) en posición preverbal (Kirsner 1979).
} 
abarca más de la mitad de los casos. Sin embargo, encontramos una variedad bastante importante de V alternativos: como se desprende de la tabla abajo, se trata de algo más de un caso de cada tres ${ }^{4}$.

\begin{tabular}{|l|c|c|c|c|c|c|c|c|}
\hline Cuadro 1 & \multicolumn{2}{|c|}{ Er staat } & \multicolumn{2}{|c|}{ Er zit } & \multicolumn{2}{|c|}{ Er ligt } & \multicolumn{2}{|c|}{ Total } \\
\hline & $\#$ & $\%$ & $\#$ & $\%$ & $\#$ & $\%$ & $\#$ & $\%$ \\
\hline Traducción literal & 2 & 1,5 & 2 & 1 & 3 & 4 & 7 & 2 \\
\hline V existencial haber & 83 & 62 & 83 & 46 & 48 & 59 & 214 & 54 \\
\hline Otros V & 41 & 30,5 & 84 & 47 & 23 & 28 & 148 & 38 \\
\hline Ausencia de V & 8 & 6 & 10 & 6 & 7 & 9 & 25 & 6 \\
\hline Total & 134 & 100 & 179 & 100 & 81 & 100 & 394 & 100 \\
\hline
\end{tabular}

\subsection{La traducción literal y el problema de la interferencia}

Antes de abordar el verdadero tema de esta contribución, dedicamos algunas palabras al fenómeno de la traducción literal. En el caso presente, esta opción le permite al traductor mantener la expresión explícita de la posición física del objeto en cuestión. Así en (7) la factura yace, o sea se encuentra en posición horizontal, mientras que en (8) el V erguirse implica la verticalidad del objeto situado en el espacio. Finalmente, en (9) los caballeros están sentados, es decir que se insiste en que se encuentran en una posición corporal específica.

(7) Een rekening van de Broeders van Sint-Vincent lag in Meerke's schoot. (VVB, 519)

La factura de los Hermanos de San Vicente yacía sobre el regazo de Mamuca.

(8) Midden op de schoorsteenmantel boven de open haard stond glanzend de Oscar die Benson ruim dertig jaar geleden in ontvangst had genomen. (DHH, 150)

En el centro de la repisa de la chimenea, encima del hogar se erguía brillante el Oscar que

Benson había recibido hacía más que treinta años.

(9) In de tanktorens zaten ridders met zwart geoliede jekkers. (VVB, 312)

En las torres blindadas de los tanques estaban sentados los caballeros con cazadoras negras untadas con grasa.

Si en los tres primeros casos el $\mathrm{V}$ español expresa la postura del referente, no es así en el último ejemplo: el texto original no implica necesariamente la posición corporal sentada, puesto que el uso de zitten en in een toren zitten ('estar (sentado) en una torre') puede indicar simplemente la presencia en el edificio, sin precisión sobre la postura concreta. Por ende, el traductor no ha mantenido la información inicial, sino que ha añadido algún matiz al texto. Esto muestra lo difícil que es para el traductor español enfrentarse a la abundancia de información detallada en cuanto a la postura en neerlandés. De hecho, parece inevitable que una traducción fiel al texto original ponga de relieve una información que en la versión original forma parte del telón de fondo.

En estos ejemplos se trata sin duda de casos de «interferencia », lo que les ha llevado a algunos lingüistas a criticar - o incluso rechazar - la metodología aquí adoptada. Sin embargo, hoy día se reconoce comúnmente que el trabajo sobre este corpus traductivo permite sacar a luz fenómenos

\footnotetext{
${ }^{4}$ Destaca también la frecuencia mayor de er zit en neerlandés para con los dos otros V de postura. Este hecho es bastante llamativo puesto que según Lemmens (2002) el V de postura más frecuente (y semánticamente más neutro) sería er staan. ¿Acaso er zit sea más apropiado en contextos resentativos? De momento dejamos este asunto para otra contribución.
} 
difíciles de vislumbrar a partir de corpus monolingües (i. a. Borin 2002, Noël 2003). De hecho, el análisis de nuestro corpus confirma que, como veremos más adelante, este método permite sacar a luz algunos aspectos inesperados dentro del español.

\subsection{Clases de verbos en español}

Como vimos en la tabla anterior, en la casi totalidad de los casos los traductores evitan precisar la postura física de los SN. En la mitad de ellos recurren al V existencial haber. Visto que solo estudiamos la traducción de los $\mathrm{V}$ de postura en un contexto presentativo, este hecho no debe extrañar. De hecho, haber es el V presentativo por excelencia en español (i. a. Suñer 1982). Se considera generalmente como un $\mathrm{V}$ monoactancial excepcional por el hecho de tener como único argumento un objeto directo (OD).

(10) Hier vlak tegenover ligt een woonschip, waarop een schipper met vrouw en kinderen huist, [...]. (HA, 60)

Justo aquí en frente hay un barco vivienda en el que viven un patrón, su mujer y sus niños.

En la otra mitad de los casos, los traductores se sirven de una gran variedad de $\mathrm{V}$ que podemos clasificar en dos categorías según su estructura argumental: V intransitivos y transitivos. Los V intransitivos son $\mathrm{V}$ monoactanciales cuyo único argumento funciona como sujeto (S). En general se trata de los llamados $\mathrm{V}$ inacusativos de existencia, aparición y localización como existir, surgir y encontrarse (cf. Levin / Rappaport Hovav 1995). Esos V son particularmente aptos para utilizarse en contextos presentativos porque su único argumento es en realidad un argumento interno que se encuentra típicamente en posición posverbal.

(11) Als de DDR opengaat ligt er toch ook een grote mogelijkheid voor West-Duitsland? (BN, 27) Si se abren las puertas de la RDA existen grandes posibilidades para la RFA ¿no?

(12) $[\ldots]$ dat ze altijd bang is dat er kortsluiting optreedt, dat er woorden in een fout kanaal zitten, zodat die niet naar buiten kunnen [...]. (DV, 182)

[...] contándome que le aterroriza un posible cortocircuito mental y que las palabras surjan por el canal equivocado y no le salgan.

(13) Rechts naast ons ligt een groot zakenpand, links een meubelmakerij; [...]. (HA, 21) A la derecha de nuestro edificio se encuentra una filial de la firma Keg, de Zaandam, y a la izquierda una ebanistería.

Los $\mathrm{V}$ transitivos, o sea $\mathrm{V}$ biactanciales con un $\mathrm{S}$ y un OD, también se prestan a la introducción de nuevos referentes en el discurso. Así, debido a la ley de un elemento nuevo por frase, el S preverbal suele contener información conocida, mientras que el OD posverbal suele aportar información nueva. Los $\mathrm{V}$ transitivos encontrados en el corpus suelen utilizarse en un sentido estático, es decir no agentivo. Obsérvense a título ilustrativo los siguientes ejemplos con tener y cubrir.

(14) Ik zou toch een keer de ringen van die drie willen zien, wat voor een speciale inscriptie er staat. (VVB, 56)

Algún día me gustaría echar un vistazo a los anillos de esas tres para ver qué inscripción especial llevan.

(15) Er lagen zalmroze kleedjes op de tafel. (GMK, 228)

Unos manteles de color rosa salmón cubrían todas las mesas.

Dentro de esta categoría destaca la frecuencia de la construcción pasiva refleja, en que encontramos entre otros algunos $\mathrm{V}$ de percepción como percibirse y observarse. Esta construcción pasiva se 
caracteriza por la ausencia de un S preverbal definido y la presencia de un sintagma nominal (SN) posverbal indefinido, por lo que se adapta particularmente a contextos presentativos.

(16) Er zat niet de minste ironie in zijn stem [...]. (DEM, 212)

En su voz no se percibía la más mínima ironía, [...].

(17) De vorige keren had ze altijd wel ergens wat zien borrelen of dampen of hadden er op tafels en kasten verschillende schaaltjes en proefbuizen met vloeistoffen gestaan. (DEM, 134)

Las veces anteriores siempre había visto borbotear o humear algo, o en las mesas y armarios se observaban diferentes bandejas y tubitos de muestras con líquidos.

Al examinar la frecuencia relativa de $\mathrm{V}$ existenciales, transitivos e intransitivos, resalta que para er staat y er ligt hay una distribución muy paralela entre el uso de las tres categorías sintácticas. Para ambos $\mathrm{V}$, haber aparece en el 60 por ciento de los casos, mientras que existe un equilibrio entre el uso de los $\mathrm{V}$ transitivos e intransitivos:

\begin{tabular}{|l|l|l|l|l|l|l|l|l|}
\hline Cuadro 2 & \multicolumn{2}{|c|}{ Er staat } & \multicolumn{2}{|c|}{ Er zit } & \multicolumn{2}{|c|}{ Er ligt } & \multicolumn{2}{|c|}{ Total } \\
\hline & $\#$ & $\%$ & $\#$ & $\%$ & $\#$ & $\%$ & $\#$ & $\%$ \\
\hline Haber & 83 & 63 & 83 & 47 & 48 & 60 & 214 & 55 \\
\hline V transitivo & 21 & 15,5 & 54 & 30 & 10 & 13 & 85 & 22 \\
\hline V intransitivo & 21 & 15,5 & 32 & 18 & 15 & 19 & 68 & 17 \\
\hline Ausencia de V & 8 & 6 & 10 & 6 & 7 & 9 & 25 & 6 \\
\hline Total & 134 & 100 & 180 & 100 & 80 & 100 & 394 & 100 \\
\hline
\end{tabular}

Salta a la vista que la traducción de er zit da lugar a otra imagen. Si bien la frecuencia del uso intransitivo no es diferente, destaca una disminución relativa del $\mathrm{V}$ haber a favor de un aumento del uso transitivo. Los siguientes datos revelan que este aumento se debe sobre todo a la frecuencia del V tener como traducción de er zit:

\begin{tabular}{|l|l|l|l|l|l|l|l|l|}
\hline Cuadro 3 & \multicolumn{2}{|c|}{ Er staat } & \multicolumn{2}{|c|}{ Er zit } & \multicolumn{3}{|c|}{ Er ligt } & \multicolumn{2}{|c|}{ Total } \\
\hline & $\#$ & $\%$ & $\#$ & $\%$ & $\#$ & $\%$ & $\#$ & $\%$ \\
\hline Haber & 83 & 63 & 83 & 47 & 48 & 60 & 214 & 55 \\
\hline Tener & 7 & 5 & 41 & 23 & 4 & 5 & 52 & 13 \\
\hline Otros & 44 & 33 & 56 & 31 & 28 & 35 & 128 & 32 \\
\hline Total & 134 & 100 & 180 & 100 & 80 & 100 & 394 & 100 \\
\hline
\end{tabular}

En otras palabras, para la traducción de er zit compiten haber y tener, mientras que para er staat y er ligt la opción con tener no es típica. Esto sugiere que er zit conlleva algún rasgo léxico-semántico que puede requerir el uso de tener o impedir la traducción por haber. A ese respecto, es interesante recordar que zitten da lugar a dos extensiones metafóricas, la inclusión (containment-zitten) y el contacto fijo (contact-zitten), ilustradas en (18) y (19) respectivamente (Lemmens 2002).

(18) Er zitten appels in de rugzak.

'Están sentadas manzanas en la mochila.' 
(19) Er zit een postzegel op de omslag.

'Está sentado un sello en el sobre.'

En la segunda parte de este artículo, nos proponemos examinar el uso de la pareja haber y tener en nuestro corpus. Nos preguntamos en particular si la predilección por tener en ciertos contextos se debe más bien a una diferencia de índole semántica o discursivo-pragmática.

\section{Análisis semántico de haber y tener, ¿dos verbos locativo-existenciales?}

\subsection{Introducción}

En la literatura se postula con gran frecuencia un acercamiento entre los V de existencia (como haber) y los de posesión (como tener) ${ }^{5}$. Se trataría en esencia de dos categorías de $\mathrm{V}$ de localización (i. $a$. Clark 1970, Lyons 1967, Freeze 1992, Heine 1997) ${ }^{6}$. Así, según esta hipótesis locativa, los V de existencia localizarían prototípicamente al OD en un lugar espacio-temporal, mientras que los $\mathrm{V}$ de posesión situarían a su OD en un espacio humano, o sea en el poseedor. En otras palabras, la diferencia entre el $\mathrm{V}$ existencial haber y el $\mathrm{V}$ posesivo tener consistiría fundamentalmente en la índole inanimada y humana de la localización respectivamente. Con haber la localización se realiza bajo la forma de un SP como (20) y con tener bajo la de un S sintáctico como en (21) .

(20) En España hay muchas lenguas.

(21) Los españoles tienen horarios peculiares.

Asimismo existe bastante consenso sobre el hecho de que, contrariamente a la locución existencial there is, el $\mathrm{V}$ to have en inglés expresa una relación particular entre el $\mathrm{S}$ y el OD. Se distingue generalmente entre cinco usos fundamentales de tener según el tipo de relación que existe entre ambos elementos: a) la posesión de un bien como en (22); b) la posesión inalienable como en (23); c) la relación parte-todo como en (24); d) la relación contenedor-contenido como en (25); e) la cadena de tópico como en (26).

(22) Sus vecinos tienen tres coches.

(23) Este gato solo tiene 3 pies.

(24) El coche tiene un parachoques nuevo.

(25) El vaso tiene vino tinto.

(26) Esta fábrica de zapatos tiene más de 200 obreros.

Sin embargo, queda mucha controversia a la hora de definir o caracterizar esta relación de manera global (cf. Partee 1999, Sæbø 2009). ¿Qué es lo que estos cinco usos tienen en común? Según Van De Putte (1983) tener expresa una relación jerárquica. Baron y Herslund (2001: 86 s.) hablan de una «relación de inclusión denotativa », mientras que para Gutiérrez-Rexach (2007) la construcción

\footnotetext{
${ }^{5}$ Para un resumen de la evolución diacrónica del uso de ambos V (como predicado y auxiliar), véase Delport (2004).

${ }^{6}$ De hecho, ciertas lenguas dan cuenta de la afinidad entre ambas estructuras en su comportamiento morfológico. Así, para decir tengo un libro el ruso dice $u$ menja est' kniga ('en mí hay libro') y el turco bende kitap ('en mí libro').

${ }^{7}$ Aunque exista mucha controversia sobre el papel sintáctico del SP locativo que suele aparecer con los V existenciales, hoy se acepta que se trata en realidad de un argumento externo, funcionando en cierta medida como el S del V (Fernández Soriano 1999, Meulleman / Roegiest 2012).
} 
existencial con have expresa un lazo «esencial» en oposición a there is que expresaría un lazo « accidental ».

Para averiguar bajo qué condiciones el español opta por tener rechazando así el $\mathrm{V}$ presentativo haber, nuestro análisis empírico se centra primero en las propiedades semánticas de la localización y del objeto situado con ambos $\mathrm{V}$, antes de focalizar el tipo de relación semántica que se predica entre los dos argumentos para cada $\mathrm{V}$.

\subsection{Análisis cuantitativo}

\subsubsection{El locativo con haber frente al $\mathrm{S}$ de tener}

En cuanto a las propiedades semánticas de la localización con haber y tener, distinguimos entre tres casos: (a) el locativo es animado, bajo la forma de un SP con haber (27) o de un S con tener (28); (b) el locativo puede ser inanimado, bajo la forma de un SP con haber (29) o de un S con tener (30); (c) haber puede prescindir de un locativo (explícito) como en (31), pero claro está que tener no puede prescindir de un S.

(27) Sólo la clara mirada de los ojos mostraba que había vida en los niños. (DEM)

(28) Los exámenes la han agotado; en la parte interior del brazo tiene un moratón: [...]. (DAZ)

(29) En las sillas a ambos lados del cabecero no había ni libros ni ropa, [...]. (DEM)

(30) [...] con Tía Nora, que encontraba que el café tenía demasiada achicoria. (VVB)

(31) El hermano Alfonso gritó que había una doble trapista esperándole. (VVB)

Conforme la hipótesis locativa, los datos de nuestro corpus muestran que haber suele ir acompañado de un SP locativo que por lo general refiere a un lugar inanimado. En cambio, con tener la preferencia por un locativo humano es menos nítida de lo que era de esperar: se trata de algo menos de la mitad de los casos ${ }^{8}$.

\begin{tabular}{|l|l|l|l|l|l|l|l|l|}
\hline Cuadro 4 & \multicolumn{2}{|l|}{ Loc/S animado } & \multicolumn{2}{l|}{ Loc/S inanimado } & \multicolumn{3}{l|}{ Sin locativo explícito } & \multicolumn{2}{|l|}{ Total } \\
\hline & $\#$ & $\%$ & $\#$ & $\%$ & $\#$ & $\%$ & $\#$ & $\%$ \\
\hline Haber & 8 & 4 & 168 & 80 & 34 & 16 & 210 & 100 \\
\hline Tener & 24 & 46 & 28 & 54 & & & 52 & 100 \\
\hline
\end{tabular}

Así, parece que en nuestro corpus tener no se comporta como un V locativo que sitúa al OD en un « sitio humano ». Sin embargo, cabe observar que cuando tener tiene un S animado, éste coocurre casi siempre con un segundo locativo, que aparece bajo la forma de un SP, lo que es muy raro en el caso de un $\mathrm{S}$ inanimado:

\begin{tabular}{|l|l|l|l|l|l|l|}
\hline Cuadro 5 & \multicolumn{2}{|l|}{ Loc } & \multicolumn{2}{l|}{ Ausencia de loc } & \multicolumn{2}{l|}{ Total } \\
\hline Tener & $\#$ & $\%$ & $\#$ & $\%$ & $\#$ & $\%$ \\
\hline S animado & 21 & 87,5 & 3 & 12,5 & 24 & 46 \\
\hline S inanimado & 2 & 7 & 26 & 93 & 28 & 54 \\
\hline
\end{tabular}

\footnotetext{
${ }^{8}$ Claro está que los porcentajes en la tabla 4 solo son representativos en cuanto traducciones de zitten en contextos presentativos.
} 
Estos SP locativos suelen ser anafóricos con el S humano y designar una parte del cuerpo (32) o alguna pieza de ropa (33), o sea entidades consideradas como de «posesión inalienable ». En los raros casos en que no aparece un SP, el OD de tener siempre indica una parte del cuerpo (como las ojeras en (34)), por lo que su localización (en este caso en la cara o debajo de los ojos) resultaría demasiado evidente para ser explicitada.

(32) Pero tienes un poco de pasta de dientes en la comisura de los labios. (DEM, 99)

(33) Hace poco tenía un fajo de billetes de veinte francos en el bolso. (VVB, 421)

(34) Gastaba una barba rojiza con la que Rex no le había visto nunca y tenía enormes ojeras que, de color azul claro, contrastaban con la pálida piel de su frente y pómelos. (DEM)

Según Freeze (1992: 581), la presencia de SP anafóricos con have solo se puede explicar si el S se analiza como una localización: «the locative subject licenses the presence of the anaphoric phrase ». En otras palabras, confirma la hipótesis de que los $\mathrm{V}$ posesivos representan un tipo de $\mathrm{V}$ de localización.

Queda, pues, de manifiesto que tanto haber como tener se comportan como V locativos en nuestro corpus. Una primera diferencia importante entre el $\mathrm{V}$ existencial y el posesivo consiste en la frecuencia de locativos de índole humana con tener, que además suelen ir acompañados por SP espaciales anafóricos.

\subsubsection{El objeto directo de haber y tener}

A fin de averiguar si el OD prototípico de haber difiere semánticamente del de tener, distinguimos entre tres casos: el OD refiere a objetos (35), a seres humanos (36) o a animales (37). Estos tres tipos de OD se ejemplifican en las siguientes frases con haber:

(35) Beck ve ahora claramente que en el pelo de Alexandra hay una ramita de árbol. (DAZ)

(36) Fuera había centenares de niños que habían aguardado toda la noche, [...]. (KIS)

(37) Porque en el sótano hay ratas. (VVB)

Del cuadro abajo, que representa los porcentajes de los tres tipos de OD por V, se desprende una predilección común por OD inanimados. En cambio, cuando se trata de un OD animado, surge una diferencia sorprendente entre ambos V: el OD de tener nunca refiere a un humano, mientras que el OD de haber no suele referir a animales.

\begin{tabular}{|l|l|l|l|l|l|l|l|l|}
\hline Cuadro 6 & \multicolumn{2}{|l|}{ OD inanimado } & \multicolumn{2}{l|}{ OD humano } & \multicolumn{2}{l|}{ OD animal } & \multicolumn{2}{|c|}{ Total } \\
\hline & $\#$ & $\%$ & $\#$ & $\%$ & $\#$ & $\%$ & $\#$ & $\%$ \\
\hline Haber & 187 & 89 & 20 & 9,5 & 3 & 1,5 & 210 & 100 \\
\hline Tener & 43 & 90 & & & 5 & 10 & 48 & 100 \\
\hline
\end{tabular}

En conclusión, nuestros datos empíricos ofrecen una imagen bastante intrigante de las propiedades semánticas de ambos $\mathrm{V}$ : a) los locativos con tener presentan una proporción mucho más importante de referencias humanas que con el V haber; y b) los OD animados suelen referir a animales con tener y a seres humanos con haber. De ser así, es de esperar que la relación semántica entre la entidad situada y el sitio en que se encuentra es fundamentalmente diferente.

3.3. El lazo semántico entre los dos argumentos de haber y tener 
Visto que en nuestro corpus el uso de tener se hace más frecuente en cuanto se trata del V neerlandés er zitten, nos preguntamos en primer lugar si y en qué medida los dos conceptos metafóricos asumidos por zitten en neerlandés, es decir la inclusión y el contacto, propician el uso de tener en español.

\subsubsection{Inclusión}

Para expresar la inclusión, el neerlandés utiliza por lo general in iets zitten ('estar sentado dentro de algo'), sin especificar la postura del objeto. En nuestro corpus español, la traducción más frecuente de este tipo de contexto es el V haber como en (38). Cuando se trata de la inclusión de un objeto dentro de otro de manera suelta, sin que el tipo de contenido sea previsible sin abrir o destapar el contenedor, haber entra en competencia con contener como en (39).

(38) Er zat geen brief in de map, enkel een kaart met data en een paar zwartwitfoto's. (DEM, 291) En la carpeta no había ningún documento, sólo una tarjeta con datos y un par de fotos en blanco y negro.

(39) Hij maakte er een open: er zaten voetzoekers in. (DZ, 121)

Abrió una: contenía buscapiés.

En cambio, cuando una entidad hace parte de otra, o sea que se trata de una relación meronímica, los traductores suelen recurrir a tener. Así, en (40) los tornillos son una parte inherente del váter, mientras que en (41) se trata del número de cigarros en una caja destinada a tal destino. Bajo esta luz, no es de extrañar que se trata frecuentemente de objetos de posesión inalienable como las partes del cuerpo (42).

(40) No sé si nuestro váter tiene tornillos, nunca lo he examinado con tanto detenimiento, pero lo haré un día de éstos. (DAZ, 210)

(41) Tiene tres cigarros, y mis amigos pintaron una calavera y firmaron en la cajetilla. (DZ, 24)

(42) En cualquier caso tenía las manos despellejados, los brazos medio descolocados y un desgarrón en la rodillera. (DZ, 272)

En otras ocasiones, tener parece utilizarse para indicar que el argumento incluido tiene un impacto en el contenedor. Este impacto es particularmente obvio en casos como (43) en que la presencia de la entidad contenida por la mezcla de varias sustancias cambia el sabor del contenedor. De la misma manera, en (44)-(46), la presencia de grasa, bultitos y moratones en el cuerpo humano conlleva un cambio fundamental en la percepción del cuerpo. Paralelamente, en los ejemplos (47) y (48) el contenedor sufre una alteración negativa que es visiblemente manifiesta en su superficie bajo la forma de un daño definitivo.

(43) Ella estaba comiéndose una rebanada de pan con mermelada de membrillo con Tía Nora, que encontraba que el café tenía demasiada achicoria. (VVB, 29)

(44) No tenía ni un gramo de grasa en las caderas y sus piernas eran largas, bonitas y musculosas. (DR, 56)

(45) Piensa en el cuerpo de su mujer, la barriga sobre la cual había colocado su mano tantas veces mientras le decía que de verdad no estaba demasiado gorda; y ese bultito que tiene en la espalda, arriba a la derecha, y que Beck insiste en que sea extirpado por un especialista. (DAZ, 151)

(46) Los exámenes la han agotado; en la parte interior del brazo tiene un moratón: la enfermera no era muy hábil con la jeringuilla. (DAZ, 19)

(47) Para Beck, su ropa era un disfraz, y ahora ese disfraz tenía rasgones; y los rasgones lo irritaban pues un disfraz desgarrado es un mal disfraz. (DAZ, 142) 
(48) Todavía hay dos países y todavía está el muro, aunque tenga agujeros. (BN, 76)

En este respecto, es particularmente reveladora la comparación con contextos parecidos en los que haber aparece con el OD agujero. De hecho, los ejemplos siguientes dejan entrever que el uso de haber nunca implica una alteración perjudicial. Así, en (49) los agujeros en los matorrales son inherentes a la planta y en (50) el agujero es natural al nacimiento de la criatura. En (51) el agujerito es esencial en el funcionamiento del juguete.

(49) En varios lugares había agujeros grandes entre los matorrales. (DR, 47)

(50) A causa del agua que el sacerdote echa por el cráneo - en el que hay un agujero que no cicatriza hasta después del bautizo, ya que si no el Espíritu Santo no podría llegar al cráneo. (VVB, 62)

(51) En el mango aplanado había un agujerito por el que se podía ver el Sacré-Cœur de París, con los detalles en tono pastel. (VVB, 287)

En breve, tener solo se utiliza cuando el contenedor resulta de algún modo afectado por la entidad incluida. Esta constatación permite explicar por qué tener solo se combina con OD animados de índole animal, contrariamente a haber que se construye tanto con animales como con humanos. De hecho, en nuestro corpus, tener suele utilizarse con bichos cuya presencia es percibida como muy negativa: el lugar en que se encuentran está concebido como apestado por su presencia. Se trata más concretamente de pulgas en un cojín (52), algunos bichos en el agua (53) y un gusanito en la comida (54). En cambio, cuando haber aparece con un OD animal, se trata simplemente de su presencia en un lugar del que se pueden quitar con relativa facilidad sin que este lugar haya sufrido daño alguno como en $(55)^{9}$.

(52) Y tenía miedo que [el cojín] tenía pulgas. (HA, 192)

(53) Pero no pudieron utilizarla [el agua] porque los americanos la analizaron primero y parece ser que tenía unos bichos. (VVB, 204)

(54) [la carne] Tenía ya un gusanito y todo. (VVB, 481)

(55) $[\ldots]$ desde el vientre que le ardía como si también allí hubiera moscas, cientos de moscas que quisieran salir en tropel al exterior. (DEM, 415)

En resumidas cuentas, los ejemplos con tener no expresan la inclusión básica en que un objeto suelto se encuentra dentro de otro. En tal caso se prefiere el uso de haber o contener. Con tener el contenedor (o sea el S) sufre una alteración fundamental por la presencia del contenido (o sea el OD). Así, el lazo entre el S y el OD de tener parece ser más estrecho del que existe entre el locativo y el OD de haber.

\subsubsection{Contacto}

Cuando entre un objeto y su localización no existe un lazo de inclusión, sino de contacto, el neerlandés utiliza op iets zitten / liggen / staan ('estar sentado / tumbado / de pie encima de algo'). La diferencia entre (56) con liggen y (57) con zitten es que con zitten las dos entidades en contacto son inseparables, mientras que con liggen sí se pueden separar. Como es de esperar a partir de nuestras observaciones anteriores, esta diferencia neerlandesa se ve reflejada en español. Así, en (56) haber se limita a establecer una simple relación locativa entre el abrigo y la piel. Al revés, en (57) tener presenta la relación entre el sello y el sobre como meronímica.

\footnotetext{
${ }^{9}$ Está claro que los humanos no suelen alterar el lugar en que se sitúan, salvo en frases como España tiene 46 millones de habitantes en que se caracteriza al país.
} 
(56) Louis vond zijn moeder, zittend op de rand van het bed in haar onderjurk, op haar knieën lag een stekelige, glinsterende bontjas. (VVB, 318)

Louis encontró a su madre sentada al borde de la cama en combinación; sobre sus rodillas había un abrigo de piel como de púas, reluciente.

(57) ,De brief aan Graaf Grisenstijn, ' zei Frans. ,Hier is hij. Maar er zit nog geen postzegel op. (DZ, 97)

(La carta para el conde Grisenstijn) Aquí está pero todavía no tiene sello.

De hecho, al estudiar las ocurrencias de contacto en el corpus resulta útil distinguir entre el contacto entre dos entidades sueltas y el que existe entre dos entidades fijadas o pegadas la una a la otra. Es más, muchas veces ni siquiera hay contacto real, sino que un objeto está fijado al lado de otro, creando una relación de pertenencia inalienable como en (58) y (59).

(58) ¿Por qué todas las ventanas de la clausura tienen barrotes, hermana Imelda? (VVB, 171)

(59) Poco después vio, en ese lado una senda lateral, pero también estaba cerrada y además tenía un letrero. $(\mathrm{DZ}, 122)$

En conclusión, el uso de tener se observa cuando la presencia del OD implica una alteración del S, o sea cuando existe un lazo de « impacto » entre ambos argumentos. En otros términos, el uso de tener no sirve para expresar la inclusión ni el contacto sino más bien el impacto.

\section{Haber vs. tener, ¿dos verbos presentativos?}

Antes de describir el comportamiento discursivo-pragmático de haber y tener en nuestro corpus, cabe recordar que ambos $\mathrm{V}$ se utilizan en construcciones fundamentalmente diferentes en cuanto a su estructura temática. Así, haber da lugar a una estructura tética introduciendo referentes en el discurso, mientras que tener expresa una relación categórica entre dos términos, el S y el OD. Bajo esta luz, es llamativo que para traducir al español la construcción tética er zit los traductores utilicen a veces construcciones categóricas con $\mathrm{V}$ biactanciales como tener. A continuación examinamos primero la capacidad de ambos $\mathrm{V}$ de introducir referentes en el discurso, antes de adentrarnos en la importancia de la presencia de un tema en las frases con tener.

\subsection{Introducción de referentes}

A fin de averiguar la medida en que haber y tener se utilizan para introducir referentes nuevos en el discurso, el cuadro abajo presenta la proporción de SN indefinidos con ambos V. Resulta que tanto para haber como para tener la presencia de un OD indefinido es más frecuente que la de un OD definido, lo que no es de extrañar ya que en la literatura se dice que ambos $\mathrm{V}$ están sometidos a la restricción de definitud (i. a. Partee 1999). Además se trata aquí de traducciones de zitten en un contexto exclusivamente presentativo.

\begin{tabular}{|l|l|l|l|l|l|l|}
\hline Cuadro 7 & \multicolumn{2}{|l|}{ OD indefinido } & \multicolumn{2}{l|}{ OD definido } & \multicolumn{2}{l|}{ Total } \\
\hline & $\#$ & $\%$ & $\#$ & $\%$ & $\#$ & $\%$ \\
\hline Haber & 208 & 99 & 2 & 1 & 210 & 100 \\
\hline Tener & 43 & 88 & 6 & 12 & 49 & 100 \\
\hline
\end{tabular}

Sin embargo, queda claro que ambos $\mathrm{V}$ no se comportan de la misma manera. En el caso de haber la presencia de un OD definido es muy excepcional, mientras que con tener se trata de una de cada diez ocurrencias. De hecho, estos datos confirman la idea de que la restricción de definitud que domina 
sobre tener no es tan estricta como en el caso de haber (Gutiérrez-Rexach 2007). En este sentido haber parece ser un $\mathrm{V}$ más prototípicamente presentativo que tener.

A este respecto, Sanz Martin (2011: 61-79) hace observar con acierto que la identificabilidad del OD de tener difiere fundamentalmente entre la construcción posesiva y la con predicación secundaria. En la construcción posesiva, que versa sobre la relación entre poseedor y poseído, el S suele proporcionar información conocida, mientras que el OD aporta información nueva, tal como se muestra en (60). En cambio, en contextos de predicación secundaria, la enunciación versa sobre el estado del OD, de ahí que tanto el $\mathrm{S}$ como el OD son portadores de información dada. La información nueva se halla exclusivamente en el predicado secundario, como se ilustra en (61):

(60) Para Beck, su ropa era un disfraz, y ahora ese disfraz tenía rasgones; [...]. (DAZ, 142)

(61) En cualquier caso tenía las manos despellejadas, los brazos medio descolocados y un desgarrón en la rodillera. (DZ, 272)

Ahora bien, en nuestro corpus encontramos todavía otro tipo de contextos en que el OD de tener es formalmente definido e pragmáticamente identificable. Así, es interesante señalar que en algunos ejemplos haber y tener aparecen en contextos semánticamente muy parecidos pero pragmáticamente diversos: en la primera ocurrencia con haber el OD es nuevo en el discurso y aparece bajo la forma indefinida, mientras que una página más adelante la construcción se repite con tener con un OD definido, ya mencionado en el discurso. Obsérvense a título ilustrativo las parejas (62)-(63) y (64)(65):

(62) Beck ve ahora claramente que en el pelo de Alexandra hay una ramita de árbol. (DAZ, 131)

(63) Sigue teniendo la ramita en el pelo. (DAZ, 132)

(64) Entre los omoplatos había una gasa blanca del tamaño de un sello de correos que se hallaba pegada a la piel con esparadrapo. (DEM, 65)

(65) En la espalda tenía la misma gasa blanca. (DEM, 66)

En la pareja (64)-(65) llama la atención que el SP espacial en la frase con haber sufra dos cambios importantes en la frase con tener: la persona se convierte en el S (elíptico) de tener y la parte del cuerpo en un SP anafórico para con el S. De hecho, a parte de que tener permite repetir una idea ya señalada en el contexto anterior, también difiere de haber por tener un $\mathrm{S}$ temático.

\subsection{Tematicidad}

Con vistas a examinar la importancia de disponer de un tema en el uso de tener, observemos ahora algunos ejemplos en que haber y tener se oponen a pesar de utilizarse en contextos presentativos (con OD nuevos) y semánticamente muy parecidos. La primera pareja (66)-(67) muestra que haber aparece en un contexto tético, mientras que el $\mathrm{S}$ de tener es temático, es decir que hay correferencia con el $\mathrm{S}$ de dijo en la proposición anterior. En la segunda pareja observamos un fenómeno parecido. En ambos ejemplos el poseedor de las lágrimas es temático, pero en (68) hay un cambio correfencial de la primera a la tercera persona, mientras que en (69) se continúa a hablar en tercera persona.

(66) Había un resplandor marrón oscuro en su piel. (DHH, 214)

(67) No dijo una palabra pero tenía un halo triunfal en la cara. (DZ, 222)

(68) - ¿Por qué he tenido que despertarme? - gimió cuando abrió los ojos un poco después. Siempre me despierto. - Había lágrimas en sus ojos. La ardilla estaba a su lado. (DGK, 68)

(69) - Soy la ardilla - susurró el elefante. La ardilla no dijo nada. ¿Qué tendría que haber dicho entonces? - preguntó el elefante. Tenía lágrimas en los ojos. La ardilla le ayudó a incorporarse 
con cuidado, le frotó un par de chichones, le enderezó la trompa y se encargó de que el elefante volviera a tener aspecto de elefante. (DGK, 91)

Ahora bien, en los ejemplos arriba, ¿cómo explicar que el traductor alterna el uso de haber con el de tener? Postulamos que el traductor recurre a tener para poder implicar a un tercer argumento. Así, en (70) el tema (en el sentido de aboutness) son los pies y no su poseedor. Al contrario, en (71) no solo los pies tienen su importancia sino también sus poseedores, lo que se ve formalmente en el hecho de que el infinitivo arrancar solo puede referir al S de tener. De la misma manera, en (73) se insiste en una relación de inclusión y nada más, mientras que en (72) se implica el poseedor del bolsillo como argumento implicado.

(70) Louis se postró a los pies atravesados y besó los dedos largos y elegantes sobre los que había cuatro gráciles ojos de gallo. (VVB, 266)

(71) Cada noche entre los dedos de los pies, teníamos unas garrapatas gordas y tenaces que, según Billie había que arrancar girando en sentido contrario al de las agujas del reloj. (HS, 14)

(72) $[\ldots]$ en voz más baja añadió: en su bolsillo izquierdo tiene algo más. (DZ, 39)

(73) Francisco le siguió con la mirada y, entre tanto, notó que, en efecto, había algo más en su bolsillo. (DZ, 39)

Esta implicación de un tercer argumento tiene sus consecuencias para la estructura narrativa. Así, en (74) se describe a Víctor, un personaje que tiene barba y ojeras. De hecho, la descripción de Víctor ya se había iniciado en la frase anterior y continúa en la oración siguiente. Al contrario, (75) no se concentra en la descripción del personaje Kapriski, sino en una sucesión de eventos en la que la descripción de sus ojos solo es relevante para describir la situación en que se encuentra. En otros términos, se trata de añadir un hecho objetivo y no de seguir tematizando como en (74).

(74) Rex miró a Victor con los ojos abiertos de par en par. No le sorprendieron sus palabras o su enfado, sino su aspecto tan cambiado. Gastaba una barba rojiza con la que Rex no le había visto nunca y tenía enormes ojeras que, de color azul claro, contrastaban con la pálida piel de su frente y pómelos. Debía de llevar una semana sin afeitar y, probablemente, durante todo ese tiempo apenas habría dormido. (DEM, 255)

(75) Pero la compañía minera no permitía su despido. Kapriski vació su vaso de un trago. Bajo sus ojos había profundas bolsas. La inflexión en su voz reapareció y me recordó el comienzo de la noche, cuando me había recogido de la estación. ¿No habría estado llorando sentado a solas en su coche? (DR, 119)

En breve, la diferencia capital entre el uso de haber y de tener es que este V permite implicar un tercer argumento que el traductor quiere tematizar. Eso podría explicar la alta frecuencia de $\mathrm{S}$ humanos con tener frente a su baja importancia con haber. De hecho, es bien sabida la tendencia universal que los $\mathrm{S}$ (y por extensión los temas) son prototípicamente humanos.

\section{Conclusión}

En neerlandés la función presentativa de introducción de un referente nuevo en el universo del discurso se expresa mediante el $\mathrm{V}$ copulativo ser, pero también mediante varios $\mathrm{V}$ de postura (staan, liggen, zitten). En la presente contribución examinamos en qué medida en español haber, el $\mathrm{V}$ presentativo por excelencia, compite con otros $\mathrm{V}$ para expresar los matices vehiculados por los $\mathrm{V}$ de postura en neerlandés. Así, averiguamos que para traducir los $\mathrm{V}$ neerlandeses staan, liggen y zitten en contextos presentativos los traductores suelen recurrir al $\mathrm{V}$ existencial haber, a varios $\mathrm{V}$ intransitivos de aparición e incluso a algunos $\mathrm{V}$ transitivos con significado estático. Estas estrategias de traducción 
se presentan cualquiera sea el $\mathrm{V}$ original en neerlandés, lo que sugiere que el español no expresa la postura de manera alguna.

En ese contexto sorprende que contrariamente a lo esperado, de los tres $\mathrm{V}$ de postura neerlandeses zitten se traduzca con menos frecuencia por haber y eso a favor del $\mathrm{V}$ tener. Un análisis semántico de ambos $\mathrm{V}$ en nuestro corpus reveló que, contrariamente al locativo que aparece con haber, el S de tener posee una incidencia importante de referentes humanos. Además, el OD de haber puede referir tanto a referentes inanimados, animales y humanos, mientras que con tener este último tipo de referente no suele presentarse. Encima observamos que tener se utiliza cuando la entidad localizada tiene algún impacto en la entidad incluyente.

Aunque diferentes, el concepto de «impacto» se acerca a ciertos usos de las dos extensiones metafóricas principales que caracterizan el uso de zitten en neerlandés: la inclusión (containmentzitten) y el contacto (contact-zitten). De hecho, cuando er zit expresa inclusión el traductor español debe expresar si se trata de una inclusión «libre» o «comprometida». En el primer caso los dos referentes incluyente e incluido son autónomos y el traductor utilizará haber o contener (como en hay un libro en la mochila = la mochila contiene un libro). En el segundo caso, el referente incluido no funciona como argumento autónomo puesto que afecta al referente incluyente. El traductor recurre a tener (como en el libro tiene 200 páginas = es un libro de 200 páginas). Este $\mathrm{V}$ expresa pues una relación muy estrecha entre los dos argumentos a tal grado que los dos elementos ya no se perciben de manera separada. Se trata de una diferencia conceptual que el neerlandés no suele expresar.

Para explicar esta constatación resulta interesante reparar en la sintaxis de haber y tener. Por un lado ambos V tienen un grado de transitividad muy bajo (Hopper / Thompson 1980). Se nota p. ej. en la imposibilidad de la transformación pasiva (*el pan fue habido / *la novia fue tenida), la ausencia del acusativo preposicional con OD humanos (hay gente buena / tiene novia) y la posibilidad del nombre escueto (hay pan / tiene novia). En otras palabras, los dos V se caracterizan por un OD incorporado en el $\mathrm{V}$ que por ende funciona en cierta medida como un $\mathrm{V}$ soporte. Por otro lado, los dos $\mathrm{V}$ difieren de manera fundamental en cuanto al número de argumentos seleccionados por el V: haber es monoactancial (si no tenemos en cuenta el complemento locativo), mientras que tener es biactancial. En breve, el OD de haber es conceptualmente autónomo, mientras que el de tener mantiene una relación estrecha o jerárquica con el $\mathrm{S}$.

Sin embargo, la comparación de usos semánticos muy parecidos de ambos $\mathrm{V}$ mostró que aunque no cabe duda de que se trata de un factor particularmente favorecedor del uso de tener, el impacto sobre la localización por parte de la entidad situada no impide la traducción por haber. Parece ser que la diferencia capital entre ambos V no es de índole semántica, sino pragmática. Así, se utiliza tener cuando se tematiza la entidad incluyente, lo que corresponde a la naturaleza inherentemente categórica del V tener y lo que podría explicar la incidencia relativamente importante de referentes humanos en la posición del S.

Gent / Brussel-Talk, diciembre de 2011

Referencias bibliográficas

Corpus

Beckman, T. (1993): Kruistocht in spijkerbroek, Rotterdam: Lemniscaat. [KIS]

Beckman, T. (1991): Cruzada en "jeans", Madrid: SM. 
Brijs, S. (2005): De Engelenmaker, Antwerpen: Atlas. [DEM]

Brijs, S. (2009): El hacedor de ángeles, Madrid: Alianza.

Brokken, J. (1991): De Regenvogel, Amsterdam: De Arbeiderspers. [DR]

Brokken, J. (2001): El pájaro de la lluvia, Barcelona: Alba.

Claus, H. (2004): De Zwaardvis, Amsterdam: De Bezige Bij. [DZV]

Claus, H. (1992): El pez espada, Barcelona: Anagrama.

Claus, H. (1992): Het verdriet van België, Amsterdam: De Bezige Bij. [VVB]

Claus, H. (1990): La pena de Bélgica, Madrid: Alfaguara.

Dorrestein, R. (2002): Een Hart van Steen, Amsterdam / Antwerpen: Contact. [HS]

Dorrestein, R. (2003): Album de Familia, Barcelona: Anagrama.

Dragt, T. (1999): De Zevensprong, Amsterdam: Leopold. [DZ]

Dragt, T. (2007): El Enigma del Séptimo Paso, Madrid: Siruela.

Frank, A. (1986): Het Achterhuis, Amsterdam: Bakker. [HA]

Frank, A. (2007): Diario, Barcelona: Debolsillo.

Grunberg, A. (2007): De Asielzoeker, Amsterdam: Nijgh \& Van Ditmar. [DAZ]

Grunberg, A. (2008): El refugiado, Barcelona: Tusquets.

Grunberg, A. (2000): De Geschiedenis van mijn Kaalheid, Breda: De Geus. [GMK]

Grunberg, A. (2004): Como me quedé calvo, Barcelona: Tusquets.

Nooteboom, C. (1990): Berlijnse Notities, Amsterdam: De Arbeiderspers. [BN]

Nooteboom, C. (1992): La desaparición del muro, crónicas alemanas, Barcelona: Península.

Nooteboom, C. (2001): De Omweg naar Santiago, Amsterdam: Atlas. [OS]

Nooteboom, C. (2001): El desvío a Santiago, Madrid: Siruela.

Palmen, C. (1996): De Vriendschap, Amsterdam: Prometheus. [DV]

Palmen, C. (1996): La Amistad, Madrid: Debate.

Tellegen, T. (1999): De Genezing Van De Krekel, Amsterdam: Querido. [DGK]

Tellegen, T. (2003): La Curación del Grillo, Madrid: Siruela.

Winter, L. (2000): De Hemel van Hollywood, Amsterdam: De Bezige Bij. [DHH]

Winter, L. (1999): El cielo de Hollywood, Barcelona: Circé.

Obras consultadas 
Ameka, F. K. / Levinson, S.C. (2007): "Introduction - The typology and semantics of locative predicates: Posturals, positionals and other beasts", en: Linguistics 45/5, 847-872.

Baron, I. / Herslund, M. (2001): Dimensions of Possession, Amsterdam / Philadelphia: Benjamins.

Borin, L. (2002): Parallel corpora, parallel worlds, Amsterdam: Rodopi.

Bull, W. (1943): "Related Functions of haber and estar", en: Modern Language Journal 27, 119-123.

Clark, E. (1970): "Locationals: a study of the relations between existential, locative and possessive constructions", en: Working papers on language universals 3, 11-37.

Conti Jiménez, C. (2005): "Existential sentences with preposed locative phrases and postverbal determinerless subjects in Spanish", en: Linguistics 46, 1079-1104.

Delport, M.-F. (2004): Deux verbes espagnols: haber et tener, Paris: Editions hispaniques.

Fernández Soriano, O. (1999): "Two types of impersonal sentences in Spanish: locative and dative subjects", en: Syntax 2, 101-140.

Freeze, R. (1992): “Existentials and other locatives”, en: Language 68, 553-595.

Gutiérrez-Rexach, J. (2007): "Beyond the (in)definiteness restriction: A unified semantics for have", en: E. Puig-Waldmuller (ed.), Proceedings of Sinn und Bedeutung 11, Barcelona: Universitat Pompeu Fabra, 291-304.

Heine, B. (1997): Possession. Cognitive sources, forces and grammaticalization, Cambridge: CUP.

Hopper, P. / Thompson S. (1980): “Transitivity in Grammar and Discourse”, en: Language 56/2, 251259.

Kirsner, R. (1979): The problem of presentative sentences in modern Dutch, Amsterdam /New York: North-Holland Publishing Company.

Kuno, S. (1972): “The position of locatives in existential sentences", en: Lingvisticae Investigationes 2, 333-378.

Lambrecht, K. (1994): Information structure and sentence form, Cambridge: CUP.

Lambrecht, K. / Polinsky, M. (1997): “Typological variation in Sentence-Focus Constructions?”, en: K. Singer / R. Eggert / G. Anderson (eds.), Proceedings of the Thirty-third Annual Meeting of the Chicago Linguistic Society. Papers from the Panels, Chicago: Chicago Linguistic Society, 189-206.

Lemmens, M. (2002): “The semantic network of Dutch posture verbs”, en: J. Newman (ed.), 103-131.

Lemmens, M. (2005): « Motion and location: toward a cognitive typology », en: G. Girard /L. Roux (eds.), Parcours linguistique. Domaine anglais, Saint-Etienne: Publications de l'Université de SaintEtienne, 223-244.

Levin, B. / Rappaport Hovav, M. (1995): Unaccusativity at the syntax-lexical semantics interface, Cambridge/Mass.: MIT Press.

Lyons, J. (1967): “A note on possessive, existential and locative sentences”, en: Foundations of Language 3, 390-396. 
Meulleman, M. / Roegiest, E. (2012): « Los locativos en la valencia de la construcción existencial española: ¿actante o circunstante? », en: ZRPh 128, 57-70.

Newman, J. (2002): The linguistics of sitting, standing, and lying, Amsterdam / Philadelphia: Benjamins.

Noël, D. (2003): “Translations as evidence for evidence for semantics: an illustration", en: Linguistics $41 / 4,757-785$.

Partee, B. (1999): “Weak NPs in HAVE-sentences”, en: J. Gerbrandy / M. Marx / M. De Rijke / Y. Venema (eds.), JFAK: Essays presented to Johan van Benthem on the occasion of his 50th birthday, Amsterdam: Amsterdam University Press, CD-ROM.

Sanz Martin, B. E. (2011): El proceso de auxiliarización del verbo tener. De la posesión al aspecto, Aguascalientes: Universidad Autónoma de Aguascalientes.

Sæbø, K. J. (2009): "Possession and pertinence: the meaning of have", en: Natural Language Semantics 17, 369-397.

Serra Borneto, C. (1996): “Liegen and stehen in German: A study in horizontality and verticality", en: E. Casad (ed.), Cognitive Linguistics in the Redwoods, Berlin: Mouton De Gruyter, 458-505.

Suñer, M. (1982): Syntax and Semantics of Spanish Presentational Sentence-Types, Washington: Georgetown University Press.

Talmy, L. (2000): Toward a cognitive semantics, Cambridge/Mass.: MIT. 